\title{
BMJ Open Protocol for 'Resilient Caregivers': a randomised trial of a resilience-based intervention for psychologically distressed partner caregivers of patients with cancer
}

\author{
Peter Genter, ${ }^{1}$ Beverley Lim Høeg (D) , ${ }^{2}$ Camilla Jensen Hamre, ${ }^{1}$ \\ Elisabeth Anne Wreford Andersen, ${ }^{3}$ Susanne Oksbjerg Dalton, ${ }^{4}$ Bjørn Ribers, ${ }^{5}$ \\ Pernille Envold Bidstrup ${ }^{2}$
}

To cite: Genter P, Høeg BL, Hamre CJ, et al. Protocol for 'Resilient Caregivers': a randomised trial of a resilience-based intervention for psychologically distressed partner caregivers of patients with cancer. BMJ Open 2021;11:e048327. doi:10.1136/ bmjopen-2020-048327

\section{- Prepublication history and} additional supplemental material for this paper are available online. To view these files, please visit the journal online (http://dx.doi.org/10.1136/ bmjopen-2020-048327).

Received 23 December 2020 Accepted 25 0ctober 2021

Check for updates

(C) Author(s) (or their employer(s)) 2021. Re-use permitted under CC BY-NC. No commercial re-use. See rights and permissions. Published by BMJ.

For numbered affiliations see end of article.

Correspondence to Dr Beverley Lim Høeg; bevlim@cancer.dk

\section{ABSTRACT}

Introduction Intimate partners of patients with cancer often experience significant distress, but there is a lack of psychological interventions that specifically target this population. 'Resilient Caregivers' is a novel resilience-based intervention for distressed partner cancer caregivers. The intervention was developed according to a resilience framework focusing on meta-reflective skills, coping strategies and value clarification. The aim of this study is to evaluate the effectiveness of this intervention in a randomised trial.

Methods and analysis Eighty participants will be invited through the Oncology Department at Herlev Hospital, Denmark and randomised to either the intervention or usual care. Participants are eligible if they are partners (married or unmarried) of patients diagnosed with cancer and experience distress ( $>4$ on the distress thermometer). 'Resilient Caregivers' consists of seven manualised group sessions (2.5 hours each), focusing on resilience in relation to being a partner caregiver of a patient with cancer. The primary outcome is symptoms of anxiety, while secondary outcomes include distress, depression, quality of life, sleep quality and resilience. Data will be collected at baseline, 3, 6 and 12 months follow-up using validated scales, and analysed using mixed models for repeated measures. Ethics and dissemination This study will follow the ethical principles in the Declaration of Helsinki and has been reviewed by the Ethics Committee of the Capital Region of Denmark (Journal no. 18055373). Written informed consent will be obtained from all participants. Results will be reported through scientific peer-reviewed journals and relevant conferences.

Trial registration number NCT04610034.

\section{BACKGROUND}

A cancer diagnosis affects not just the patient, but also the lives of close family and friends, who often serve as informal caregivers providing unpaid care. ${ }^{1}$ In many cases, the primary informal caregiver will be the spouse or intimate partner of the patient with cancer
Strengths and limitations of this study

- This is the first resilience-based intervention that specifically targets vulnerable partner caregivers of patients with cancer.

- This is a randomised trial with long-term follow-up (12 months) and with adequate power to detect an effect in the primary outcome of anxiety.

- The study takes place in a counselling setting with potential for national implementation.

- Caregivers were involved in the development of the intervention and materials for trial participants.

- Due to nature of this study, only data analysts can be blinded, but not participants and providers.

and increasing research indicates that the health and well-being of partner caregivers of patients with cancer are adversely affected by this role. ${ }^{12}$ Furthermore, caregivers often suppress their own needs in order to focus on the needs of their ill partner. ${ }^{3}$ As a result, partners of patients with cancer have been shown to experience physical and psychological impairment due to high levels of distress, ${ }^{2}{ }^{4}$ and have an increased risk of depression and anxiety, ${ }^{56}$ insomnia and sleep problems, ${ }^{57}$ alcohol misuse ${ }^{8}$ and even early death. ${ }^{910}$ This indicates a pressing need for interventions targeting distressed caregivers and for improving their ability to cope with the stresses of caring for a partner with cancer.

Previous interventions for partner caregivers of patients with cancer

Previous interventions have largely targeted informal caregivers in general, which include family members and friends. ${ }^{11}$ We found three systematic reviews ${ }^{11-13}$ and two metaanalyses $^{1415}$ that summarised the literature 
on interventions for informal caregivers of patients with cancer. The largest review included 50 trials from 2010 to $2016^{12}$ and showed that the majority of interventions focused on information and skills related to the physical care of the patient, with less focus on caregiver self-care. Furthermore, out of the 50 studies, only $54 \%(n=39)$ of the studies referred to a theoretical framework and only $34 \%$ $(\mathrm{n}=24)$ addressed intervention fidelity. ${ }^{12}$ The two metaanalyses showed inconsistent results, probably due to methodological differences. The first meta-analysis included 29 randomised trials and showed small to medium treatment effects of the interventions (psychoeducational, skills training and therapeutic counselling) on improving caregiver burden, distress and anxiety, self-efficacy and ability to cope. ${ }^{14}$ The second meta-analysis specifically assessed the effectiveness of cognitive-behavioural therapies (CBT) in 36 trials and showed a small, statistically significant effect of CBT interventions when evaluating randomised and non-randomised trials together. ${ }^{15}$ However, this effect became non-significant when only randomised trials were evaluated, and the authors concluded that future interventions should move beyond traditional CBT methods. This may be because traditional CBT methods focusing on changing maladaptive beliefs may not be as relevant in a caregiver setting, where distressing thoughts are often not rooted in cognitive distortions but rather real threats from a chronic or fatal illness. The same author group subsequently published a randomised trial of emotion regulation therapy (ERT) for cancer caregivers that showed medium to large reductions in psychological distress, worry and caregiver burden. ${ }^{16}$ ERT developed from CBT but focuses on managing perseverative negative thinking (worry and rumination) using emotion regulation skills supported by mindfulness practices.

We found only one systematic review of psychological interventions specifically targeting the well-being of partners of patients with cancer, which identified six randomised trials and three pre-post intervention studies. ${ }^{17}$ Only three randomised trials reported positive intervention effects on the outcomes of emotional distress, social support, post-traumatic growth and coping. However, most of the studies were limited by low participation rates, small sample sizes (average sample size was 43) and a lack of long-term follow-up, with only two studies having follow-up measurements at 6 months. Many studies also did not screen for distressed participants making it difficult to detect any significant improvement in study outcomes, which may explain the small or non-existent effect sizes found in the meta-analyses cited. Thus, work is still needed to develop more effective interventions for distressed partner caregivers of patients with cancer that are based on newer therapeutic approaches and tested in designs with sufficient power and follow-up. An approach based on a framework that goes beyond traditional CBT methods, with an added focus on caregiver needs and self-care, may improve on some of the limitations of existing interventions and lead to improved efficacy.

\section{Resilience as a therapeutic approach}

Resilience is commonly referred to as the ability to recover or sustain well-being after an adverse life event, and represents a paradigm shift in psychology where there is increased focus on well-being and protective factors instead of solely on mental illness and risk factors. ${ }^{18} 19$ A cancer diagnosis in a partner may be considered an adverse life event, as one is faced not only with the potential death of a loved-one, but also with the responsibility of caregiving and assuming the daily tasks and roles of the ill partner. ${ }^{111}$ A resilience-based approach, which focuses on regaining balance and building psychological capacity, may thus be highly relevant in developing an intervention to support distressed partner caregivers. One systematic review of 25 randomised trials of resilience interventions found favourable effects for enhancing resilience, improving stress and reducing depressive symptoms among a wide range of populations including soldiers, employees, students and physicians. ${ }^{20}$ Among cancer survivors, a handful of resilience-based interventions have shown promising results for outcomes such as resilience, stress and anxiety ${ }^{21-24}$ and a few observational studies have shown an association between resilience among caregivers of adult patients with cancer and caregiver outcomes such as self-reported health status, anxiety and depression. ${ }^{325}{ }^{26}$ However, no study has yet been carried out for a resilience-based intervention for cancer caregivers.

\section{Rationale for an integrative approach for targeting resilience in partner caregivers of patients with cancer}

Personal capacities that have been shown to be correlated to resilience include meaning and purpose in life, active coping, optimism and cognitive flexibility. ${ }^{27} 28$ Many of these factors have been shown to be modifiable through newer psychological approaches, often categorised as the 'third wave' of $\mathrm{CBT}^{29}$ While traditional CBT examines the content of thoughts or feelings and its rationality, third wave approaches focus more on a person's relationship to thoughts or emotions, and whether these ways of thinking and feeling are helpful in the context of the person's life. Examples of third-wave approaches include metacognitive therapy (MCT), acceptance and commitment therapy (ACT) and dialectic behavioural therapy (DBT).

MCT targets metacognitive beliefs, for example, the belief that worrying will help to solve a problem, through techniques such as attention training that helps patients reflect on, and relate differently to, these beliefs. ${ }^{30}$ In ACT, the goal is to help the individual clarify their personal values and act based on them, while learning to be present with what life brings, including difficult feelings. ${ }^{31}$ Similarly, DBT also teaches ways to cope with distress and regulate difficult emotions. ${ }^{32}$ However, in DBT, there is a dialectical focus, for example, not just on acceptance but also on change, and on balancing the often dual and opposing nature of thoughts, feelings and coping strategies. ${ }^{32}$ All three therapeutic approaches outlined above 


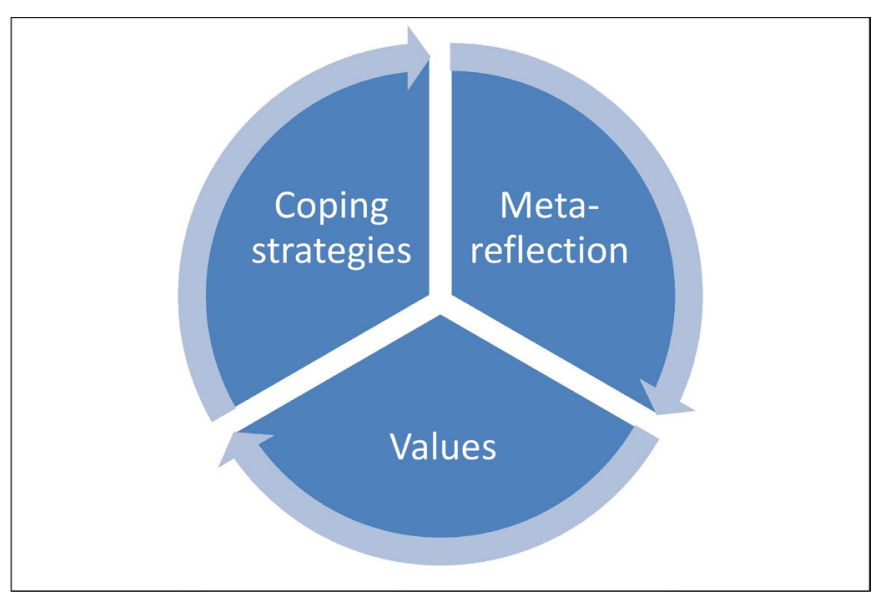

Figure 1 Proposed central components of the resilience process: the meta-reflective skill of evaluating and choosing values-based coping strategies.

build on elements of mindfulness or non-judgemental awareness, which may be especially helpful in the cancer setting, where uncertainty and distressful thoughts and feelings are normal responses that need to be acknowledged. We propose that an integrative approach based on meta-reflection, valued living and personalised coping strategies are fundamental to promoting resilience in the partner caregiver of patients with cancer.

\section{Proposed theoretical framework}

Few resilience interventions have based their work on a defined model of resilience. ${ }^{28}$ In this study, we define resilience as the dynamic process of recovering or maintaining well-being after a cancer diagnosis in a partner. This process is based on the meta-reflective skill of stepping back, evaluating and choosing from different coping strategies in order to achieve a values-based outcome for the individual (figure 1). Below, we briefly outline our approach with regards to each proposed component central to our definition of resilience.

\section{Coping strategies}

We categorise coping strategies as belonging to the following polarities: acceptance strategies versus change strategies, strategies to manage perceived negative emotions versus strategies to manage perceived positive emotions, and strategies based on social connections vs strategies strengthening individuality/independence. Within our framework, resilience is enhanced by being able to navigate and choose from different coping strategies best suited to a particular context.

\section{Meta-reflection}

In order to navigate and choose from dialectical coping strategies, one must first be able to step back and be able to see one's behaviour and situation from a larger perspective. We define 'meta-reflection' as this ability to reflect on one's thoughts and actions, in order to evaluate whether a given coping strategy is helpful for one's situation, as well as whether it contributes to living a life based on what is important to the individual.

\section{Valued living}

The final component in the proposed model is the clarification of personal values and the ability to navigate and act based on what is important in one's life. Within our framework, resilience is enhanced when a person is able to think and cope in ways that he or she finds meaningful and are aligned with what he or she finds valuable and important in life. Thus, within our model, resilience is not based on the ability to use 'good or 'bad' strategies, but rather on being able to flexibly choose strategies that can bring an outcome that is of value to the individual. Based on the theoretical framework outlined above, we developed a resilience-based intervention for partner caregivers of patients with cancer.

\section{Pilot study}

A pilot study was carried out from November 2018 to January 2019. We recruited six cancer caregivers through the Danish Cancer Society's counselling centre and website, who received the entire programme led by PG and completed questionnaires before and after the intervention. Participants signed informed consent forms regarding their participation in the study and as user contributors to help evaluate the programme. Their contributions are provided in more detail in the Patient and public involvement section below. Focus group interviews showed high acceptability.

Descriptive analyses based on five participants who completed all questionnaire showed pre-post intervention reductions in symptoms of depression and anxiety, an increase in resilience and self-efficacy, but no change in caregiver burden.

\section{Aims and hypotheses}

In this current study, we aim to evaluate the short-term and long-term effectiveness of the 'Resilient Caregivers' intervention for improving outcomes among distressed partner caregivers of patients with cancer. We hypothesise that compared with participants in a control group, participants in the intervention group will show reduced symptoms of anxiety, depression and distress, and improved quality of life, sleep quality and resilience on completion of the intervention, and that improvements in outcomes will remain in follow-up of up to 12 months. We further aim to examine potential mechanisms of change underpinning any effect of the intervention, thereby providing initial validation of the proposed theoretical framework for this resilience-based approach. We hypothesise that the effect of the intervention in reducing symptoms of depression, anxiety and stress will be mediated by improvements in resilience, meta-reflective skills and valued living.

\section{METHODS}

\section{Design}

This randomised trial was planned according to guidelines for conducting resilience intervention studies as 
proposed by Chmitorz et $a l^{28}$ and according to the Standard Protocol Items Recommendations for Intervention Trials guidelines for clinical trial protocols. ${ }^{3435}$ Enrolment started in April 2021, with an estimated study completion date of March 2023.

\section{Setting and participants}

The intervention will take place at the Danish Cancer Society's counselling centre in Herlev, Denmark and participants will be invited through the Department of Oncology at Herlev Hospital. At the first treatment appointment (which takes place within 2 weeks of diagnosis), patients are often accompanied by a partner and a nurse will hand out an invitation package containing a brochure about the study, an informed consent form and a brief screening questionnaire with oral information about the study. Unaccompanied patients who have partners will be asked to give the invitation package to their partner. An English language example of the consent form is included in online supplemental materials. The form also included a data privacy policy section (not shown in the example) informing participants of the legal details regarding collection of their data, their rights to access this data and who to call if they wish to report a complaint.

Partners interested in participating in the trial are invited to return the consent form and screening questionnaire using the enclosed stamped envelope, after which the project coordinator (BLH) will contact them by phone. Participants who fulfil the following criteria (based on self-reported answers on the screening questionnaire) are eligible and will receive the baseline questionnaire:

1. Are a partner (including same-sex partners, married and unmarried) to an adult patient with cancer who has non-terminal cancer (eg, has been diagnosed with stage I-III/non-metastatic cancer or has an expected survival of $>6$ months).

2. Experience distress ( $>4$ on the distress thermometer).

3. Can read and write Danish and are willing to participant in all aspects of this study.

4. Are not patients with cancer themselves.

5. Have no untreated psychiatric ailments, active substance abuse or other conditions that can affect participation in a weekly group intervention.

\section{Randomisation and allocation sequence generation}

On completion of the baseline questionnaire, participants will be randomised (1:1) to either the intervention arm or control arm according to a computer-generated list in blocks of 2 or 4 , stratified by age $(<50 / \geq 50)$ and sex (male/female), to ensure a balanced number of participants in both arms, and avoid unequal distribution of participants by age or sex. In this study, automatic stratification by sex is based on the last digit of the participant's Danish social security number (males are given an odd number and females an even number) which all Danish citizens are automatically assigned at birth. In Denmark, a person can legally change gender and receive a new number reflecting this change, but the current system only allow categories as either male or female. To take into account other gender identities, we have added a self-reported gender variable to the baseline questionnaire that allows participants to self-report their gender identity. We will not exclude any participant based on gender.

Randomisation will be carried out using REDCap, ${ }^{36}$ a secure web-based application for managing research data and allocation is concealed from the project coordinator until a participant is assigned. Participants will be informed of the group allocation by telephone. Due to the nature of the intervention, blinding is only possible for data analysts but not for participants and intervention providers.

\section{Intervention and control groups}

Participants assigned to the intervention group will be invited to attend the 'Resilient Caregivers' programme. The programme is a manualised seven-session programme in closed groups aimed specifically at improving the caregiver's ability to cope with the stresses of being a partner to a patient with cancer. Each weekly session lasts for approximately two-and-a-half hours and consists of psychoeducation, the sharing of experiences, group exercises and individual homework. Sessions 1-3 focus on the caregiver, sessions 4 and 5 focus on the relationships between the caregiver and the patient with cancer and social support networks, respectively, while session six focuses on resilience in relation to self-care and care for the ill partner (table 1). Session 7 is a booster session 1 month after the end of session 6 in order to follow up on the intervention and allow participants to reflect on the benefits and challenges of the programme. The intervention will be carried out by trained psychologists/therapists.

Participants assigned to the control group will receive care as usual, which in this case implies no systematic psychological support. We chose usual care as the comparator in order to maintain the ecological validity of this study, as this is the current 'real-world' context for cancer caregivers in Denmark. For ethical reasons and to encourage participation and decrease attrition, participants in the control group will be sent a personalised package by post containing a thank you letter explaining the importance of their role in the trial and brochures regarding existing support available at the Danish Cancer Society counselling centres. Regardless of group allocation, participants are free to seek out other sources of support, such as through their general practitioner or a private therapist.

\section{Patient and public involvement}

Partner caregivers were involved in the development of trial components in conjunction with the pilot study mentioned above. After the intervention, a focus group interview was carried out by $\mathrm{BLH}$ and $\mathrm{CJH}$ to obtain participant perspectives on the format of the programme, 
Table 1 Overview of the resilient caregivers programme

\begin{tabular}{|c|c|c|c|}
\hline Session & Resilience areas & Goals & Main therapeutic techniques \\
\hline $\begin{array}{l}\text { Session } 1 \\
\text { Cancer and the great } \\
\text { balancing act }\end{array}$ & Coping strategies & $\begin{array}{l}\text { 1. Awareness of dilemmas related to cancer } \\
\text { 2. Increased clarity regarding the balance } \\
\text { between stresses and resources, and } \\
\text { coping strategies with adverse effects } \\
\text { 3. Clarification of what the caregiver wishes } \\
\text { to achieve from this course }\end{array}$ & $\begin{array}{l}\text { Goal clarification } \\
\text { Psychoeducation about resilience } \\
\text { Identification of current coping strategies } \\
\text { Reflections on the self and life with cancer using } \\
\text { pictures etc } \\
\text { Homework: 'Taking one's temperature'. Daily } \\
\text { recording of mood and reactions to specific } \\
\text { situations }\end{array}$ \\
\hline $\begin{array}{l}\text { Session } 4 \\
\text { Life with a partner } \\
\text { with cancer }\end{array}$ & $\begin{array}{l}\text { Coping strategies, } \\
\text { meta-reflection } \\
\text { and values in } \\
\text { the couple's } \\
\text { relationship }\end{array}$ & $\begin{array}{l}\text { 1. Awareness of helpful and unhelpful } \\
\text { patterns in the relationship } \\
\text { 2. Strengthened ability to shift strategies } \\
\text { when a pattern is unhelpful } \\
\text { 3. Awareness of - and identification of- } \\
\text { important values in the relationship }\end{array}$ & $\begin{array}{l}\text { Psychoeducation: relationship and } \\
\text { communication during illness } \\
\text { Reflection exercises on cancer and personal } \\
\text { values in the relationship, and changing coping } \\
\text { strategies in relation to the partner } \\
\text { Homework: What values do you want to focus on } \\
\text { in your relationship? }\end{array}$ \\
\hline $\begin{array}{l}\text { Session } 5 \\
\text { Social support } \\
\text { networks }\end{array}$ & $\begin{array}{l}\text { Coping strategies, } \\
\text { meta-reflection and } \\
\text { values related to } \\
\text { social networks }\end{array}$ & $\begin{array}{l}\text { 1. Awareness of social network and the } \\
\text { possibility for support from others } \\
\text { 2. Awareness of behavioural patterns } \\
\text { related to social support networks } \\
\text { 3. Ability to shift strategies to draw on } \\
\text { support and resources from the network } \\
\text { when needed }\end{array}$ & $\begin{array}{l}\text { Psychoeducation: the importance of social } \\
\text { support networks } \\
\text { Exercises to enhance relationship skills, map } \\
\text { out current social network and identify current } \\
\text { strategies in relation to this network } \\
\text { Tool: The app 'Sammenhold' from the Danish } \\
\text { Cancer Society (a tool to coordinate help among } \\
\text { family/friends of the patient with cancer) } \\
\text { Homework: Changing strategy in one's close } \\
\text { relationship in order to receive more support }\end{array}$ \\
\hline $\begin{array}{l}\text { Session } 6 \\
\text { Resilience through } \\
\text { self-care as a } \\
\text { caregiver }\end{array}$ & $\begin{array}{l}\text { Coping strategies, } \\
\text { meta-reflection and } \\
\text { values in self-care } \\
\text { and caring for an ill } \\
\text { partner }\end{array}$ & $\begin{array}{l}\text { 1. Increased compassion towards oneself } \\
\text { and one's partner } \\
\text { 2. Awareness of coping patterns and } \\
\text { alternative ways of coping } \\
\text { 3. Setting values-based goals as a cancer } \\
\text { caregiver }\end{array}$ & $\begin{array}{l}\text { Psychoeducation: Self-care when life is difficult } \\
\text { and resilience and the importance of balance in } \\
\text { life } \\
\text { Guided reflection exercises: how can you best } \\
\text { support yourself and your partner? } \\
\text { Rounding up: Achievements from this } \\
\text { programme? }\end{array}$ \\
\hline $\begin{array}{l}\text { Session } 7 \\
\text { (Booster session) }\end{array}$ & All areas & $\begin{array}{l}\text { 1. Recap all the areas covered in the } \\
\text { programme } \\
\text { 2. Allow group members to reconnect with } \\
\text { each other }\end{array}$ & $\begin{array}{l}\text { Facilitated structured discussions based on the } \\
\text { techniques in the programme }\end{array}$ \\
\hline
\end{tabular}

the content of each session, the facilitated exercises and homework, and the questionnaire. The interview lasted 2 hours and was audiotaped. The feedback received was used to revise programme slides and materials to its current form and content. Based on feedback that the questionnaire was repetitive and lengthy, we substituted measures with validated short-form versions where possible. Three caregivers were later involved in developing the invitation brochure to ensure clarity and comprehension. Caregivers will also be involved in disseminating study results to the general public.

\section{Data collection and management}

Data will be collected through electronic questionnaires, thus reducing missing data (with the possibility for a paper version) at baseline, after completion of the programme (approximately 3 months from randomisation) and again at 6-month and 12-month follow-up. The measures used 
Table 2 Outcomes and measures used in the questionnaire

\begin{tabular}{|c|c|c|}
\hline Outcome & Measure & Description \\
\hline Demographic information & Developed for the study & Age, gender, education, job \\
\hline Anxiety & Generalised Anxiety Disorder- $7^{37}$ & Range 0-21; higher scores=more symptoms \\
\hline Psychological distress & $\begin{array}{l}\text { Distress Thermometer }{ }^{42} \text { (without problem } \\
\text { list) }\end{array}$ & Range $0-10$; higher scores=higher distress \\
\hline Depression & Patient Health Questionnaire- $9^{43}$ & Range 0-27; higher scores=more symptoms \\
\hline Quality of life & WHO-5 Well-being Index ${ }^{44}$ & Range $0-25$; higher scores=better quality of life \\
\hline Resilience & Connor-Davidson Resilience Scale-10 1845 & Range 0-40; higher scores=greater resilience \\
\hline Stress & Perceived Stress Scale-10 46 & $\begin{array}{l}\text { Range 0-40; higher scores=higher perceived } \\
\text { stress }\end{array}$ \\
\hline $\begin{array}{l}\text { Meta-reflection (rumination/ } \\
\text { worry and coping) }\end{array}$ & Cognitive Attentional Syndrome Scale- $1^{3047}$ & $\begin{array}{l}\text { Range } 0-100 \text {; higher scores=worse rumination/ } \\
\text { worry and coping }\end{array}$ \\
\hline Valued living & $\begin{array}{l}\text { Valuing Questionnaire-items from the } \\
\text { 'Obstruction' subscale }\end{array}$ & $\begin{array}{l}\text { Range 0-30; higher scores=more interference } \\
\text { with living consistently with one's values }\end{array}$ \\
\hline Sleep & Pittsburg Sleep Quality Index ${ }^{49}$ & Range 0-21; higher scores=worse sleep quality \\
\hline Social support & $\begin{array}{l}\text { Adapted from the modified Medical } \\
\text { Outcomes Study Social Support Survey- } \\
\text { three items from the 'Emotional support' } \\
\text { subscale }^{50}\end{array}$ & Range 0-12; higher scores=more social support \\
\hline Use of professional support & Developed for the study & $\begin{array}{l}\text { Participants will be asked if they had received } \\
\text { any professional support in relation to their } \\
\text { partner's cancer diagnosis, for example, from a } \\
\text { psychologist, support group, telephone chatline, } \\
\text { doctor, nurse, other. }\end{array}$ \\
\hline $\begin{array}{l}\text { Satisfaction with programme } \\
\text { (intervention group only) }\end{array}$ & Developed for the study & $\begin{array}{l}\text { Participants will be asked to evaluate the } \\
\text { content of each session, the delivery and the } \\
\text { programme as a whole }\end{array}$ \\
\hline
\end{tabular}

to assess trial outcomes are listed in table 2. The time schedule of enrolment, interventions and assessments ${ }^{34}$ are presented in table 3. Participants will receive personalised e-mails with a secure link to the relevant questionnaires and will receive an email reminder after 1 week, and a telephone reminder after 2 weeks, if they do not respond. Data will be collected and managed using the electronic platform REDCap ${ }^{36}$ hosted on secure servers at the Danish Cancer Society. Access to data will be restricted to authorised investigators.

\section{Fidelity and adherence}

Fidelity to the treatment protocol will be assessed using a checklist listing the components of each session as laid out in the intervention manual. The therapist will be asked to complete the checklist following each session. All sessions will be audiotaped and a random selection of $50 \%$ of recordings will be assessed by the research team using the checklist. Adherence will be assessed via attendance and completion of review forms at the start of each session concerning the application of the homework exercises.

\section{Sample size and power calculations}

We calculated power simulated from a linear mixed model with a random subject effect, in a set up with four measurements (baseline and three follow-up measurements), and two groups (intervention, control) with no difference at baseline on the primary outcome of anxiety (online supplemental material 1). We plan to include 80 participants to obtain at least $80 \%$ power $(95 \%$ certainty between $75 \%$ and $85 \%$ ) for detecting a minimum difference of -3 points between intervention and control groups at 6-month and 12-month follow-up as measured by the Generalised Anxiety Disorder scale (online supplemental material 1). ${ }^{37}$ The calculations were carried out using $\mathrm{R}$ package simr with an assumed significance level of $0.05 .^{38}$

\section{Statistical analysis}

Descriptive statistics will be used to present the characteristics of the study participants and assess whether the randomisation succeeded in controlling for baseline imbalances. If needed, we will control for any baseline imbalances that may influence our outcomes, such as age, gender and education. We will report on the gender identity of the final sample, including any gender minority groups. Our primary outcome is changes in symptoms of anxiety, while secondary outcomes include changes in symptoms of depression, distress, stress, quality of life and sleep quality. Based on our theoretical model, we 
Table 3 Schedule of enrolment, interventions and assessments

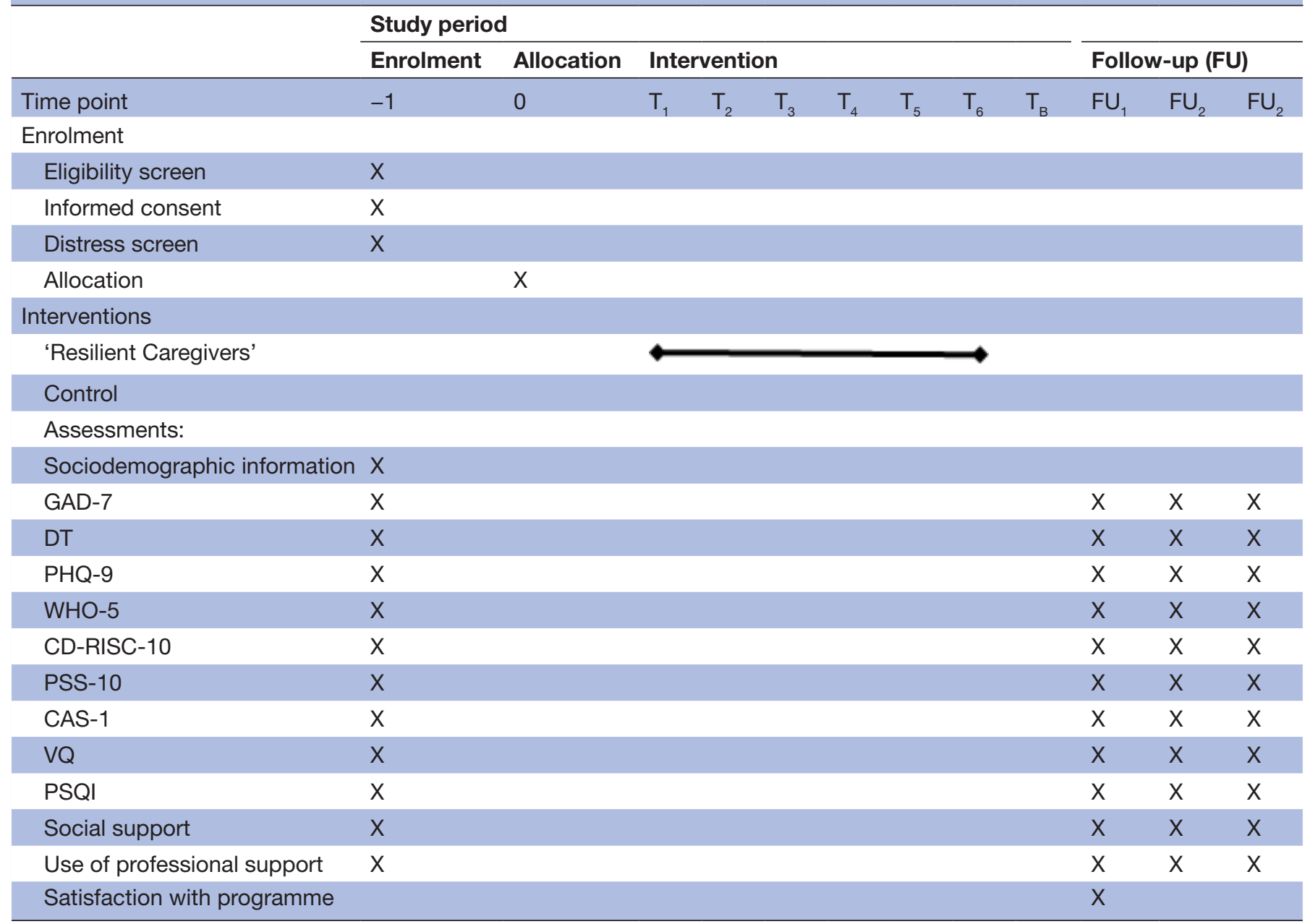

$\mathrm{T}_{1}-\mathrm{T}_{6}$ sessions 1-6; $\mathrm{T}_{\mathrm{B}}$ Booster session; $\mathrm{FU}_{1}, \mathrm{FU}_{2}, \mathrm{FU}_{3} 3,6$ and 12 months follow-up, respectively; General Self-Efficacy Scale.

CAS-1, Cognitive Attentional Syndrome Scale; CD-RISC-10, Connor-Davidson Resilience Scale; DT, Distress Thermometer; GAD-7,

Generalised Anxiety Disorder; PHQ-9, Patient Health Questionnaire; PSQI, The Pittsburgh Sleep Quality Index; PSS, Perceived Stress Scale;

VQ, Valuing Questionnaire; WHO-5, WHO 5-item Well-being Index.

also identified resilience, meta-reflective skill and valued living as potential mechanisms that may be investigated as mediators of an intervention effect on the outcomes described above.

Random effects mixed models accounting for the correlations between repeated measurements for each participant will be used to assess the effects of the intervention on completion, and at 6 and 12 months follow-up. Supplementary analyses adjusted for patient cancer type, caregiver social support and use of professional support as potential confounders will also be carried out. We also plan to assess intervention effects for subgroups of participants by carrying out interaction analyses with variables that may moderate treatment effects, such as sociodemographic and psychological characteristics of the partners or clinical characteristics of the patients. We will carry out intention-to-treat analysis and multiple imputation will be used to handle missing data.

To investigate the potential mediating role of resilience, meta-reflective skill and valued living, we will carry out exploratory analysis using structural equation models (SEM) to estimate direct and indirect associations between the intervention, outcomes and mediating variables. ${ }^{39}$ Estimates from SEM give an indication of the extent to which a variable mediates an intervention effect, thus providing evidence for its mechanistic role. ${ }^{39}$ Fidelity and adherence assessments will be summarised descriptively.

\section{Ethical considerations}

This study will follow the ethical principles in the Declaration of Helsinki and has been reviewed by the Ethics Committee of the Capital Region of Denmark (Journal no. 18055373). The need for ethical approval was waived, as it was not considered a medical science study with a clinical experimental setting. Written informed consent will be obtained from all participants prior to the study. Confidentiality will be upheld under the code of conduct for psychologists. At each group session, a check-in with participants will be carried out and any issues raised will be documented. 
In the event of a participant developing symptoms of severe psychological distress indicative of psychiatric treatment, we will advise the participant to make an appointment with their general practitioner for a referral.

Participants have the right to withdraw from the study at any time without reason and are able to contact the personnel at the counselling centre at any time, during and after the trial, regardless of whether they are in the intervention group or control group. There will be no data monitoring committee as the intervention is a psychological supportive care intervention and no medical adverse events are expected. There are no known conditions that may be expected to lead to the trial's termination. Items from the WHO Trial Registration Data Set are provided in online supplemental table 1 . This trial is also registered at the Danish Cancer Society Research Center's internal research projects register (No. 2020-DCRC-0031) under the European Union's General Data Protection Regulation.

\section{Dissemination plan}

We will report and present the scientific results of this study in accordance to the Consolidated Standards of Reporting Trials statement ${ }^{40}$ in relevant peer-reviewed journals and at scientific conferences. Authorship will follow the criteria recommended by the International Committee of Medical Journal Editors (ICMJE) in the Vancouver Protocol. ${ }^{41}$ We will also communicate trial results to participants and the general public though relevant platforms. The protocol of this study is fully available to the public through registration in ClinicalTrials.gov and this publication. Statistical codes will be available on request.

\section{Protocol amendments}

The protocol of this study may not be modified without approval from the primary investigator. Any important modifications will be communicated to all relevant parties (ie, coinvestigators, ethical committee, etc) and updated in the trial registry by the project coordinator.

\section{DISCUSSION}

To the best of our knowledge, the 'Resilient Caregivers' randomised trial is the first resilient-based intervention targeting caregivers of patients with cancer. By targeting the enhancement of resilience, this programme aims to improve the physical and psychological well-being of this important population, through the treatment and prevention of debilitating conditions such as anxiety and depression. As partner caregivers provide important support to patients with cancer, enhancing caregiver outcomes may also be expected to improve patient outcomes.

The strengths of this trial include the randomised design, the systematic invitation of participants through a major hospital and the use of an electronic data capture system to reduce missing data and collect/manage data securely. Another strength was the involvement of partner caregivers in testing the intervention and providing feedback on the feasibility and acceptability of the programme, materials and questionnaires. This trial is also adequately powered and includes long-term follow-up of up to 12 months, which is important in psychological trials in order to capture the potential effects from cognitive and behavioural adjustments, which take time to shift.

Limitations include the lack of blinding among participants and providers, which is not possible due to the nature of this trial. Another potential limitation may be attrition in questionnaire responses, especially in the control group. We will use personalised email contact with all participants to promote retention and encourage them to complete as many follow-up assessments as possible, while participants in the control group will receive a thank you letter explaining the important role of the control group in the research design. As the participants in this study are self-referred, the lack of generalisability of this study's results to the cancer caregiver population in general, and minority populations in particular, cannot be ruled out. However, we know that caregivers who seek psychological help are often those who experience a high level of distress. By screening for and including distressed participants, we expect our results to be valid for the population of cancer caregivers who seek psychological support. Finally, this study is only powered to detect changes in the primary outcome. Additional analyses, such as the use of SEM to investigate potential mechanisms of change, are therefore explorative and can only provide preliminary results. Future studies with adequate power will be needed to extend the evidence base.

We are the first to describe a theoretical formulation of resilience as applied to the large population of cancer caregivers. Findings from this trial are expected to contribute new knowledge to the understanding of the mechanisms underlying resilience processes, including the cognitions and behaviours that promote resilience in the face of stressful life events. The development of the 'Resilient Caregivers' manual based on a theoretical framework also means that mental health professionals may be easily trained to deliver this programme in an efficient manner, as it comprehensively covers important components in a relatively short period of time. The manualised group format supports cost-effective implementation in a range of healthcare settings. If shown to be efficacious, the novel 'Resilient Caregivers' programme has the potential to improve the lives of both partners and patients with cancer.

\section{PROTOCOL VERSION}

Date: 5 October 2021. This is the second revised version after peer review (V.3.0).

\section{Author affiliations}

${ }^{1}$ Herlev Cancer Counseling Center, Danish Cancer Society, Copenhagen, Denmark ${ }^{2}$ Psychological Aspects of Cancer Research Group, Danish Cancer Society Research Center, Copenhagen, Denmark

${ }^{3}$ Statistics and Data Analysis, Danish Cancer Society Research Center, Copenhagen, Denmark 
${ }^{4}$ Survivorship and Inequality in Cancer Research Group, Danish Cancer Society Research Center, Copenhagen, Denmark

${ }^{5}$ Department for the Study of Culture, University of Southern Denmark, Odense, Denmark

Acknowledgements The authors thank all the partner cancer caregivers who have contributed to the development of this study. We further thank Pernille Slebsager and Bo Andreassen Rix, MD from the Danish Cancer Society for feedback on the counseling setting and caregivers' needs, as well as Lisa Sengeløv, MD and the dedicated nurses at the Department of Oncology, Herlev Hospital, who will be assisting in the invitation of participants in this study. We also thank our peer reviewers for providing valuable feedback that helped us improve this protocol.

Contributors PG, BLH and PEB conceptualised the study design and PEB is the principle investigator. $P G$ developed the intervention and program manual together with CJH, BLH and PEB. PG is also the supervising psychologist on this trial. EAWA is supervising statistician and provided statistical support, while SOD and BR provided overall advice and content for the study protocol. BLH drafted the manuscript and is also the trial coordinator. All authors contributed to the writing and approval of the final manuscript before submission.

Funding This work is supported by the Danish Cancer Society's Psychosocial Research Committee fund (Grant number R260-A15145-19-S3 and R296-A1700619).

Disclaimer The funding body has not been involved in the study design and will not be involved in in data collection, management, analysis, and interpretation of data; writing of the report; and the decision to submit the report for publication.

Competing interests None declared.

Patient consent for publication Not applicable.

Provenance and peer review Not commissioned; externally peer reviewed.

Supplemental material This content has been supplied by the author(s). It has not been vetted by BMJ Publishing Group Limited (BMJ) and may not have been peer-reviewed. Any opinions or recommendations discussed are solely those of the author(s) and are not endorsed by BMJ. BMJ disclaims all liability and responsibility arising from any reliance placed on the content. Where the content includes any translated material, BMJ does not warrant the accuracy and reliability of the translations (including but not limited to local regulations, clinical guidelines, terminology, drug names and drug dosages), and is not responsible for any error and/or omissions arising from translation and adaptation or otherwise.

Open access This is an open access article distributed in accordance with the Creative Commons Attribution Non Commercial (CC BY-NC 4.0) license, which permits others to distribute, remix, adapt, build upon this work non-commercially, and license their derivative works on different terms, provided the original work is properly cited, appropriate credit is given, any changes made indicated, and the use is non-commercial. See: http://creativecommons.org/licenses/by-nc/4.0/.

ORCID iD

Beverley Lim Høeg http://orcid.org/0000-0002-9946-2429

\section{REFERENCES}

1 Kent EE, Rowland JH, Northouse L, et al. Caring for caregivers and patients: research and clinical priorities for informal cancer caregiving. Cancer 2016;122:1987-95.

2 Braun M, Mikulincer M, Rydall A, et al. Hidden morbidity in cancer: spouse caregivers. J Clin Oncol 2007;25:4829-34.

3 Hwang IC, Kim YS, Lee YJ, et al. Factors associated with caregivers resilience in a terminal cancer care setting. Am J Hosp Palliat Care 2018;35:677-83.

4 Bevans M, Sternberg EM. Caregiving burden, stress, and health effects among family caregivers of adult cancer patients. JAMA 2012;307:398-403.

5 Jansson MRN, von Heymann-Horan A, Rasmussen BK, et al. Risk for use of antidepressants, anxiolytics, and hypnotics in partners of glioma patients-a nationwide study covering 19 years of prescriptions. Psychooncology 2018;27:1930-6.

6 Price MA, Butow PN, Costa DSJ, et al. Prevalence and predictors of anxiety and depression in women with invasive ovarian cancer and their caregivers. Med J Aust 2010;193:S52-7.

7 Hearson B, McClement S. Sleep disturbance in family caregivers of patients with advanced cancer. Int J Palliat Nurs 2007;13:495-501.
8 Stenberg U, Ruland CM, Miaskowski C. Review of the literature on the effects of caring for a patient with cancer. Psychooncology 2010;19:1013-25.

9 Christakis NA, Allison PD. Mortality after the hospitalization of a spouse. N Engl J Med 2006;354:719-30.

10 Nakaya N, Saito-Nakaya K, Bidstrup PE, et al. All-Cause mortality among men whose cohabiting partner has been diagnosed with cancer. Epidemiology 2013;24:96-9.

11 Applebaum AJ, Breitbart W. Care for the cancer caregiver: a systematic review. Palliat Support Care 2013;11:231-52.

12 Ferrell $B$, Wittenberg E. A review of family caregiving intervention trials in oncology. CA Cancer J Clin 2017;67:318-25.

13 Frambes D, Given B, Lehto R, et al. Informal caregivers of cancer patients: review of interventions, care activities, and outcomes. West J Nurs Res 2018;40:1069-97.

14 Northouse LL, Katapodi MC, Song L, et al. Interventions with family caregivers of cancer patients: meta-analysis of randomized trials. $C A$ Cancer J Clin 2010;60:n/a.

15 O'Toole MS, Zachariae R, Renna ME, et al. Cognitive behavioral therapies for informal caregivers of patients with cancer and cancer survivors: a systematic review and meta-analysis. Psychooncology 2017;26:428-37.

16 O'Toole MS, Mennin DS, Applebaum A, et al. A randomized controlled trial of emotion regulation therapy for psychologically distressed caregivers of cancer patients. JNCI Cancer Spectr 2020;4:pkz074.

17 Kleine A-K, Hallensleben N, Mehnert A, et al. Psychological interventions targeting partners of cancer patients: a systematic review. Crit Rev Oncol Hematol 2019;140:52-66.

18 Connor KM, Davidson JRT. Development of a new resilience scale: the connor-davidson resilience scale (CD-RISC). Depress Anxiety 2003;18:76-82

19 Zautra AJ, Hall JS, Murray KE. Resilence. a new definition of health for people and communities. In: Handbook of adult resilience. New York: The Guilford Press, 2010.

20 Leppin AL, Bora PR, Tilburt JC, et al. The efficacy of resiliency training programs: a systematic review and meta-analysis of randomized trials. PLoS One 2014;9:e111420.

21 Loprinzi CE, Prasad K, Schroeder DR, et al. Stress management and resilience training (smart) program to decrease stress and enhance resilience among breast cancer survivors: a pilot randomized clinical trial. Clin Breast Cancer 2011;11:364-8.

22 Üzar-Özçetin YS, Hiçdurmaz D. Effects of an empowerment program on resilience and posttraumatic growth levels of cancer survivors: a randomized controlled feasibility trial. Cancer Nurs 2019;42:E1-13.

23 Rosenberg AR, Bradford MC, McCauley E, et al. Promoting resilience in adolescents and young adults with cancer: results from the prism randomized controlled trial. Cancer 2018;124:3909-17.

24 Eicher M, Ribi K, Senn-Dubey C, et al. Interprofessional, psychosocial intervention to facilitate resilience and reduce supportive care needs for patients with cancer: results of a noncomparative, randomized phase II trial. Psychooncology 2018;27:1833-9.

25 Simpson GK, Dall'Armi L, Roydhouse JK, et al. Does resilience mediate carer distress after head and neck cancer? Cancer Nurs 2015;38:E30-6.

26 Lim J-won, Shon E-jung, Paek M, et al. The dyadic effects of coping and resilience on psychological distress for cancer survivor couples. Supportive Care in Cancer 2014;22:3209-17.

27 Helmreich I, Chmitorz A, König J. Psychological interventions for resilience enhancement in adults. Cochrane Database Syst Rev 2017;2017:CD012527.

28 Chmitorz A, Kunzler A, Helmreich I, et al. Intervention studies to foster resilience - A systematic review and proposal for a resilience framework in future intervention studies. Clin Psychol Rev 2018;59:78-100.

29 Hayes SC, Hofmann SG. The third wave of cognitive behavioral therapy and the rise of process-based care. World Psychiatry 2017;16:245-6

30 Wells A. Metacognitive therapy for anxiety and depression. New York: Guilford Press, 2011.

31 Hayes SC, Luoma JB, Bond FW, et al. Acceptance and commitment therapy: model, processes and outcomes. Behav Res Ther 2006;44:1-25.

32 Linehan MM. Cognitive-behavioral treatment of borderline personality disorder. Guilford Publications, 2018.

33 Chapman AL. Dialectical behavior therapy: current indications and unique elements. Psychiatry 2006;3:62-8.

34 Chan A-W, Tetzlaff JM, Altman DG, et al. Spirit 2013 statement: defining standard protocol items for clinical trials. Ann Intern Med 2013;158:200-7. 
35 Chan A-W, Tetzlaff JM, Gøtzsche PC, et al. Spirit 2013 explanation and elaboration: guidance for protocols of clinical trials. $B M J$ 2013;346:e7586.

36 Harris PA, Taylor R, Thielke R, et al. Research electronic data capture (redcap)--a metadata-driven methodology and workflow process for providing translational research informatics support. J Biomed Inform 2009;42:377-81.

37 Spitzer RL, Kroenke K, Williams JBW, et al. A brief measure for assessing generalized anxiety disorder: the GAD-7. Arch Intern Med 2006;166:1092-7.

38 Green P, MacLeod CJ. SIMR : an R package for power analysis of generalized linear mixed models by simulation. Methods Ecol Evol 2016;7:493-8.

39 Kaplan D. Structural equation modeling: foundations and extensions. 2 edn. Thousand Oaks, California: Sage, 2009

40 Schulz KF, Altman DG, Moher D, et al. Consort 2010 statement: updated guidelines for reporting parallel group randomised trials. BMJ 2010;340:c332-c32.

41 ICMJE. Defining the role of authors and contributors, 2020. Available: http://www.icmje.org/recommendations/browse/roles-andresponsibilities/defining-the-role-of-authors-and-contributors.html [Accessed 1 Feb 2020].

42 Zwahlen D, Hagenbuch N, Carley Ml, et al. Screening cancer patients' families with the distress thermometer (dT): a validation study. Psychooncology 2008;17:959-66.
43 Kroenke K, Spitzer RL, Williams JB. The PHQ-9: validity of a brief depression severity measure. J Gen Intern Med 2001;16:606-13.

44 Topp CW, Østergaard SD, Søndergaard S, et al. The WHO-5 well-being index: a systematic review of the literature. Psychother Psychosom 2015;84:167-76.

45 Campbell-Sills L, Stein MB. Psychometric analysis and refinement of the connor-davidson resilience scale (CD-RISC): validation of a 10-item measure of resilience. J Trauma Stress 2007;20:1019-28.

46 Cohen S, Kamarck T, Mermelstein R. A global measure of perceived stress. J Health Soc Behav 1983;24:385-96.

47 Kowalski J, Dragan M. Cognitive-attentional syndrome - the psychometric properties of the CAS-1 and multi-measure CASbased clinical diagnosis. Compr Psychiatry 2019;91:13-21.

48 Smout M, Davies M, Burns N, et al. Development of the Valuing questionnaire (VQ). J Contextual Behav Sci 2014;3:164-72.

49 Buysse DJ, Reynolds CF, Monk TH, et al. The Pittsburgh sleep quality index: a new instrument for psychiatric practice and research. Psychiatry Res 1989;28:193-213.

50 Moser A, Stuck AE, Silliman RA, et al. The eight-item modified medical outcomes study social support survey: psychometric evaluation showed excellent performance. J Clin Epidemiol 2012;65:1107-16. 